microsphere templating for fabricating macroporous $\mathrm{CaCu}_{3} \mathrm{Ti}_{4} \mathrm{O}_{12}$ (CCTO) thin films using pulsed laser deposition (PLD) on poly(methyl methacrylate) (PMMA) templated substrates. The polymer microspheres are removed by thermal decomposition, and the residual inorganic films have unique morphologies comprising hollow hemispheres of the inorganic material with large surface areas and surface-tovolume ratios. I.-D. Kim, A. Rothschild, and co-workers reported the preparation of the CCTO thin films, as well as gas-sensitivity measurements for possible sensor applications, in the February issue of Nano Letters (DOI: 10.1021/nl051965p; p. 193).

Given its extraordinarily high dielectric constant and nonlinear $I-V$ characteristics, CCTO was expected to make an excellent sensor when interacting with reactive gases. To prepare the CCTO films, an aqueous solution of $800-\mathrm{nm}$ diameter PMMA microspheres was dripped onto $\mathrm{Al}_{2} \mathrm{O}_{3}$ substrates which had interdigitated $\mathrm{Pt}$ electrode arrays and then dried overnight. CCTO films were then deposited onto the microsphere-templated substrate using PLD. The samples were calcined in air at $800^{\circ} \mathrm{C}$ for several hours to remove the PMMA microspheres. The resultant intact films were $80-90 \mathrm{~nm}$ thick and consisted of a monolayer of hollow CCTO hemispheres with $~ 800$-nm diameters, as shown in Figure 1. Good chemical bonding between the film and substrate was observed. X-ray diffraction measurements showed that the films were singlephase polycrystalline CCTO.

A network of voids between the film and substrate allowed easy gas access to the internal surfaces of the hemispheres. The specific surface area was twice as large and the surface area four times as large as films on untreated substrates due to the hemispherical morphology and the voids - very favorable for gas sensor applications. Electrical property measurements demonstrated highly nonlinear $I-V$ characteristics with strong thermal activation. The macroporous CCTO films were tested for sensitivity to $\mathrm{H}_{2}, \mathrm{CO}$, and $\mathrm{CH}_{4}$ gases between $200-400^{\circ} \mathrm{C}$ and were found to be very sensitive to $\mathrm{H}_{2}$. In contrast, the sensitivity of films deposited on untreated substrates to $\mathrm{H}_{2}$ was negligible. The large surface area clearly promoted interactions with the gas while the relatively smaller interfacial area reduced the adverse effects of the interface between the film and the substrate. The limit of detection was determined to be about $55 \mathrm{ppm}$ at a temperature of $300^{\circ} \mathrm{C}$, comparable to the detection limit of commonly used commercial Taguchi $\mathrm{H}_{2}$ sensors. The responsivity of

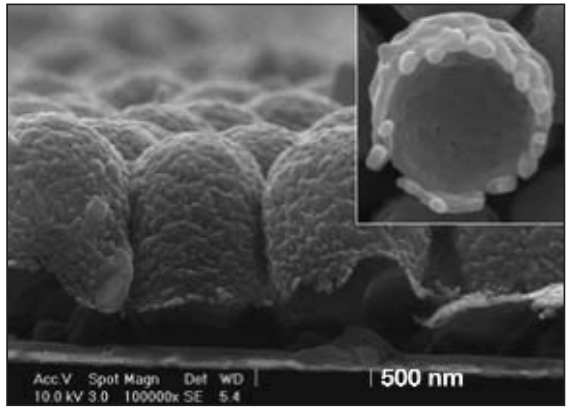

Figure 1. Scanning electron micrograph shows a cross-sectional view of the macroporous $\mathrm{CaCu}_{3} \mathrm{Ti}_{4} \mathrm{O}_{12}$ (CCTO) film formed following calcination at $800^{\circ} \mathrm{C}$. The inset shows one hollow CCTO hemisphere. (Courtesy of Avner Rothschild, MIT.)

the macroporous films to $\mathrm{H}_{2}$ was also found to be reversible.

The researchers said that in view of the simplicity of this approach, which can be applied to a wide range of materials and deposition techniques, and the remarkable improved performance, this processing strategy holds promise for chemical sensors, fuel cells, catalysts, and other electrochemical devices for energy conversion, environmental protection, and other applications. The technique has the ability to control parameters such as porosity, composition, and film thickness as well as form multilayered structures of compounds with complex chemistries and therefore is very versatile.

The research team's future plans include extending this work to other materials commonly used for gas sensing, such as $\mathrm{SnO}_{2}, \mathrm{TiO}_{2}$, and $\mathrm{ZnO}$, and examining the role of processing conditions on microsphere morphology and correspondingly on the sensing properties. The researchers will also investigate the negligible responsivity of the macroporous films to gaseous $\mathrm{CO}$ and $\mathrm{CH}_{4}$.

Gopal RAO

\section{CdSe/CdS QD-LEDs Fabricated Using a Thermally Polymerized Hole-Transport Layer}

By spin-coating CdSe/CdS quantum dot solutions onto thermally cross-linkable hole-transport layers (HTLs), a team of researchers at the University of Washington fabricated multilayer nanocrystal quantum dot light-emitting diodes (QD-LEDs) with external quantum efficiencies (EQEs) of $\sim 0.8 \%$ that exhibit spectrally pure QD emission. This method enables researchers to incorporate high-quality QD layers of varied thickness into devices with multiple solution-processed organic hole-transport layers and no organic background emission.

In the March issue of Nano Letters (DOI: 10.1021/nl052417e; p. 463), the research team, led by A.K.-Y. Jen and D.S. Ginger, reported progress in the fabrication of efficient CdSe/CdS QD-LEDs. The researchers first spin-coated the cross-linkable HTL, polystyrene- $N, N^{\prime}$-diphenyl- $N, N^{\prime}$ bis(4-n-butylphenyl)-(1,1'-biphenyl)-4,4' diamine-perfluorocyclobutane (PS-TPDPFCB), onto a plasma-cleaned indium tin oxide (ITO) slide and then heated it in nitrogen to activate the cross-linking process in the HTL. After cross-linking the PS-TPD-PFCB, the researchers spin-coated a layer of $\mathrm{CdSe} / \mathrm{CdS}$ QDs (average diameter, $4.6 \mathrm{~nm}$ ) from a chloroform solution onto the HTL. They then deposited the electron transport layer, 1,3,5-tris $(N$ phenylbenzimidazol-2-yl)benzene (TPBI), on the QD layer by thermal evaporation. They completed the devices by thermal evaporation of the back electrode $(\mathrm{Ca} / \mathrm{Ag})$.

The thermally cross-linked HTLs possess a high glass-transition temperature $\left(T_{\mathrm{g}}\right.$ $\sim 150^{\circ} \mathrm{C}$ ) and are solvent-resistant, factors which make it easy to add additional device layers such as emissive QDs by solution processing. Using atomic force microscopy (AFM), the researchers found that the surface of the polymerized PSTPD-PFCB HTL is very smooth and provides a compatible hydrophobic surface for the formation of uniform and pinholefree layers of QDs over comparatively large areas. The researchers compared the QD-LEDs with different CdSe/CdS QD coverage in the active region. They observed that the device efficiency increases with increasing QD coverage until one monolayer (ML) of dots has been deposited; then it decreases rapidly to $0.1 \%$ when the QD layer is more than $3 \mathrm{ML}$ thick. The researchers also observed a substantial decrease in EQE with increasing current density. They attributed these phenomena to slow dot-dot charge transport and a carrier imbalance or charge-induced quenching, respectively. The researchers found they could further improve the EQE of the LEDs by using a second (cross-linkable) HTL on top of the PS-TPD-PFCB layer. The EQE of these QD-LEDs is improved to $0.8 \%$ at a brightness of $100 \mathrm{~cd} / \mathrm{m}^{2}$ and the maximum brightness is improved to more than $1000 \mathrm{~cd} / \mathrm{m}^{2}$. The researchers suggested that optimizing the energy levels and better balancing carrier transport and injection into the QD emissive layer may further improve the performance of these QD-LEDs.

TAO XU 